See discussions, stats, and author profiles for this publication at: https://www.researchgate.net/publication/226416605

\title{
Comparison of AM-FM Features with Standard Features for the Classification of Surface Electromyographic Signals
}

Chapter · January 2010
DOI: $10.1007 / 978-3-642-13039-7 \_1$

\section{CITATIONS}

2

5 authors, including:

Christodoulos I. Christodoulou

University of Cyprus

53 PUBLICATIONS 1,230 CITATIONS

SEE PROFILE

A. Víctor Murray

4. UTEC - Universidad de Ingeniería y Tecnología (Peru)

76 PUBLICATIONS 722 CITATIONS

SEE PROFILE
READS

37

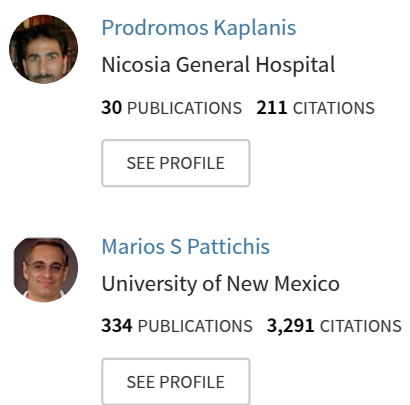

Some of the authors of this publication are also working on these related projects:

Project Linked 2 Safety View project

Project Dynamically Reconfigurable Architecture Systems for Time-Varying Image Constraints (DRASTIC) View project 


\title{
Comparison of AM-FM Features with Standard Features for the Classification of Surface Electromyographic Signals
}

\author{
C.I. Christodoulou', P.A. Kaplanis' 1 , V. Murray ${ }^{2}$, M.S. Pattichis ${ }^{2}$, C.S. Pattichis ${ }^{1}$ \\ ${ }^{1}$ Department of Computer Science, University of Cyprus, Nicosia, Cyprus \\ ${ }^{2}$ Department of Electrical and Computer Engineering, University of New Mexico, Albuquerque, USA
}

\begin{abstract}
In this work AM-FM features extracted from surface electromyographic (SEMG) signals were compared with standard time and frequency domain features, for the classification of neuromuscular disorders at different force levels. SEMG signals were recorded from a total of $\mathbf{4 0}$ subjects: 20 normal and 20 abnormal cases, at $10 \%, 30 \%, 50 \%, 70 \%$ and $100 \%$ of maximum voluntary contraction (MVC), from the biceps brachii muscle. For the classification, three classifiers were used: (i) the statistical K-nearest neighbour (KNN), (ii) the neural self-organizing map (SOM) and (iii) the neural support vector machine (SVM). For all classifiers the leaveone-out methodology was implemented for the classification of the SEMG signals into normal or pathogenic. The test results reached a classification success rate of $77 \%$ for the AM-FM features whereas standard features failed to provide any meaningful results on the given dataset.
\end{abstract}

Keywords- SEMG, AM-FM, classification

\section{INTRODUCTION}

The electromyographic (EMG) examination provides important information for the assessment of neuromuscular disorders and is generally carried out using needle electrodes. Surface electrodes and the acquisition of surface EMG signals provide a non-invasive alternative to needle EMG for the detection of neuromuscular disorders. At present a surface detected signal is preferred only for obtaining "global" information about the time and/or intensity of superficial muscle activation [1].

Time and frequency features have been extensively used in EMG signal classification [2]-[6]. Using needle EMG, Abel et al. [3] used turned analysis and small signals segments to obtain $75 \%$ classification rate for 45 cases $(12$ normal, 18 myopathy, and 15 neuropathy patients). However authors concluded that the classification methods used, did not offer better results than the interference pattern analysis and could not match the diagnostic success of an experienced clinician. Christodoulou et al. [4] developed a modular neural networks system where multiple features extracted from needle EMG signals were fed into multiple classifiers to yield $87.5 \%$ classification rate in 38 cases $(12$ normal, 13 myopathy, and 13 motor neuron disease cases).
Abou-Chadi et al [5] compared three neural networks systems for surface EMG classification. Unsupervised techniques gave $80 \%$ correct classification when tested on 10 cases (5 myopathy and 5 normal) selected from a pool of 28 cases. Recordings were performed for 5 seconds at $50 \%$ MVC. Abou-Chadi et al [5] reached the conclusion that when SEMG is properly processed, it may provide the physician with a diagnostic assisting tool. Also for surface EMG, Kaplanis [6] reached a correct classification score of $82.9 \%$ on 111 cases (91 normal and 20 abnormal cases). One may comment that this result may be misleading due to a higher number of control subjects as compared with pathogenic cases, however normalisation based on the number of subjects for each group during the classification process was performed.

For the classification of surface EMG signals, we presented an earlier study on the use of AmplitudeModulation Frequency-Modulation (AM-FM) features in [7]. In the current paper, we compare the performance of the AM-FM features against time and frequency features.

We provide a description of the data acquisition process in Section II. In section III we describe the extraction algorithms for the standard time and frequency features and the AM-FM features. In Section IV, we present results using three different classifiers: (i) the statistical K-nearest neighbour (KNN) classifier, (ii) the neural self-organizing map (SOM) and (iii) and the neural support vector machine (SVM). We give the results in Section V and provide concluding remarks in Section VI.

\section{MATERIAL AND DATA ACQUisition}

Surface EMG recordings were acquired from 20 control subjects (NOR) and 20 neuromuscular cases (11 myopathy and 9 neuropathy cases). Patients referred were first examined and diagnosed by their physician and were divided according to the general type of neuromuscular disorder (myopathy or neuropathy). The data were collected at a special Electromyography / Electroencephalography / Evoked Potential (EMG/EEG/EP) lab at the Department of Clinical Neurophysiology at the Cyprus Institute of Neurology and Genetics, Nicosia, Cyprus [6]. The Nicolet Viking IV electromyography a two-channel amplifier used

P.D. Bamidis and N. Pallikarakis (Eds.): MEDICON 2010, IFMBE Proceedings 29, pp. $69-72,2010$. www.springerlink.com 
unit was fully electrically isolated to IEC 601-1 and BSS 5724, Part 1 Type BF. The input impedance of the system, Zin was stated to be $>1000 \mathrm{M} \Omega$. Through the system the low and high frequency values for recording were set at 20 and $500 \mathrm{~Hz}$ respectively.

A calibrated force measurement system, with a total weight of $40 \mathrm{~kg}$ was placed at the foot end of a couch, used for the subjects to lie down. The weights were lifted via a strap placed at the subjects' wrist and connected to the system through a force transducer, which was connected directly to a calibration circuit. The subject was required to pull at maximum voluntary contraction (MVC) three times with an interval of two minutes in between. A note of the MVC was made on the oscilloscope with a red tape. Recordings were made at five different force levels, i.e. at $10 \%, 30 \%, 50 \%, 70 \%$ and $100 \%$ of MVC from the biceps brachii muscle generated under isometric voluntary contraction (IVC).

\section{FEATURE EXTRACTION}

\section{A. Standard Features}

For each surface EMG (SEMG) epoch, at each force level, the following parameters were measured in the time domain:

1. Turns per second $(t / s)$ : Number of slope reversals separated from the previous one and the following turn by an amplitude difference greater than $20 \mu \mathrm{V}$.

2. Zero crossings per second $(z / c)$ : Defined as the number of sign reversals exceeding a threshold of $20 \mu \mathrm{V}$.

For each $512 \mathrm{~ms}$ epoch, the average power spectrum (PS) curve was computed by taking the FFT of 512 points, with $25 \%$ overlap segments. The following parameters were computed from the power spectrum curve:

3. Median frequency: Frequency dividing the area under the PS curve into two equal parts.

4. Mean frequency

5. Maximum frequency

6. Total power: Calculated as the total area under the PS curve, with values reported in $\mathrm{nV}^{2} / \mathrm{Hz}$. Logarithmic units were taken due to the large spread of values recorded.

\section{Maximum power}

The above seven features were normalized before use by subtracting their mean value and dividing with their standard deviation.

\section{B. AM-FM features}

Amplitude-modulation frequency-modulation (AM-FM) models can be used to characterize non-stationary signal behavior [8], [9]. Using a multi-scale filter bank, for any given signal $f(k)$, we compute a one-dimensional, single scale analytic signal, as given by [8]:

$$
f_{A S}(k)=f(k)+j H\{f(k)\}
$$

where $H\{$.$\} denotes the Hilbert transform operator. We$ estimate the instantaneous amplitude (IA), the instantaneous phase (IP) and the instantaneous frequency (IF) of the signal using

$$
\begin{aligned}
& a(k)=\left|f_{A S}(k)\right| \\
& \varphi(k)=\arctan \left(\frac{\operatorname{imag}\left(f_{A S}(k)\right)}{\operatorname{real}\left(f_{A S}(k)\right)}\right) \\
& \varphi_{k}(k)=\frac{\partial \varphi(k)}{\partial k} \cong \frac{1}{n} \arccos \left(\frac{f_{A S}(k+n)+f_{A S}(k-n)}{2 f_{A S}(k)}\right)
\end{aligned}
$$

where in (4), $n$ is a variable displacement from 1 to 4 , based on the argument that provides the minimum condition number to arcos function.

From the two generated estimates, the histograms for 32 equal width bins were computed and were used as input feature sets for classification. The histograms were further normalized by division of the histogram with the number of SEMG signal points in order to alleviate any bias due to different signals lengths. Figure 1 shows a sample of SEMG signal from a normal subject and its corresponding AM-FM histograms (only shown 1000 samples points for visibility).
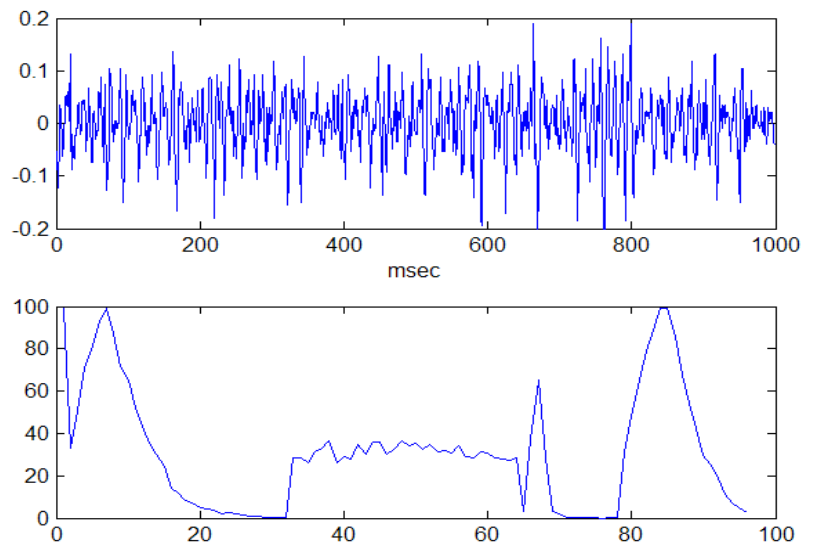

Fig. 1. Sample of SEMG signal from a normal subject at $100 \%$ force level and its corresponding AM-FM histograms. First 32 samples show the histogram of the instantaneous amplitude. This is followed by the histogram of the instantaneous phase and the instantaneous frequency.

\section{Classification}

The seven standard features and the three AM-FM histograms (i.e. 96 bins) were used as two different feature vectors and were inputted into three classifiers. The leaveone-out methodology was applied where for each input pattern to be classified all the remaining patterns were used as the training set. The average of all classifications scores was the final score. This made the classification procedure 
independent of bootstrap sets and the results more robust and reliable.

\section{A. The KNN Classifier}

The statistical k-nearest neighbor (KNN) classifier [10] was used for the classification of the SEMG signals using the leave-one-out methodology. In the KNN algorithm, in order to classify a new input pattern, its $k$ nearest neighbors from the training set were identified. The new pattern was classified to the most frequent class among its neighbors based on a similarity measure that is usually the Euclidean distance. In this work the KNN system was implemented for several values of $k(k=1,3,5,7,9,11,13$ and 15$)$ and it was tested using for input the two different feature vectors at the different force levels.

\section{B. The SOM Classifier}

The SOM was chosen because it is an unsupervised learning algorithm where the input patterns are freely distributed over the output node matrix [11]. The weights are adapted without supervision in such a way, so that the density distribution of the input data is preserved and represented on the output nodes. This mapping of similar input patterns to output nodes, which are close to each other, represents a discretisation of the input space, allowing a visualization of the distribution of the input data. The output nodes are usually ordered in a two dimensional grid and at the end of the training phase, the output nodes are labeled with the class of the majority of the input patterns of the training set, assigned to each node.

In the evaluation phase, a new input pattern was assigned to the winning output node with the weight vector closest to the new input vector. In order to classify the new input pattern, the majority of the labels of the output nodes in an $R x R$ neighborhood window centered at the winning node, were considered. The number of the input patterns in the neighborhood window for the two classes $m=\{1,2\}$, (1=normal, $2=$ pathogenic), was computed as:

$$
S N_{m}=\sum_{i=1}^{L} W_{i} N_{m i}
$$

where $L$ is the number of the output nodes in the $R \times R$ neighborhood window with $L=R^{2}$ (e.g. $L=9$ using a $3 \times 3$ window), and $N_{m i}$ is the number of the training patterns of the class $m$ assigned to the output node $i$. $W_{i}=1 /\left(2 d_{i}\right)$, is a weighting factor based on the distance $d_{i}$ of the output node $i$ to the winning output node. $W_{i}$ gives the output nodes near to the winning output node a greater weight than the ones farther away (e.g. in a $3 \times 3$ window, for the winning node $W=1$, for the four nodes perpendicular to the winning node $W_{i}=0.5$ and for the four nodes diagonally located $W_{i}$ $=0.3536$, etc). The evaluation input pattern was classified to the class $m$ of the $S N_{m}$ with the greatest value, as normal or pathogenic.

\section{The SVM Classifier}

The Support Vector Machine (SVM) was also used for developing classification models for the problem. The method is initially based on a nonlinear mapping of the initial data set using a function $\varphi($.) and then the identification of a hyperplane which is able to achieve the separation of two categories of data. Details about the implementation of the SVM algorithm used can be found in [12]. The SVM network was implemented using Gaussian Radial Basis Function (RBF) kernels; this was decided, as the rest of the kernel functions could not achieve satisfactory results.

\section{Combining}

From each subject, five feature vectors were calculated one for each force level and inputted to the classifiers. The five classification outputs per subject were further combined using majority voting, i.e. the subject was assigned to the class where the majority of the five individual SEMG signals per force level were assigned. This was done in order to get a final and more reliable estimate of the classification result, since as it was shown in [4], modular neural networks system enhanced the diagnostic performance of the individual classifiers making the whole system more robust and reliable.

\section{RESUlts}

Surface EMG recordings from 20 control subjects (NOR) and 20 neuromuscular subjects (11 myopathy (MYO) and 9 neuropathy (NEURO)) were recorded at 10\%, 30\%, 50\%, $70 \%$ and $100 \%$ of maximum voluntary contraction (MVC), from the biceps brachii muscle. For each SEMG recording two different feature vectors were extracted as described above (i) seven standard features from time and frequency domain (ii) the AM-FM features instantaneous amplitude (IA), instantaneous phase (IP), and the instantaneous frequency (IF). The IA, IP, IF were normalized by the signal length in order to alleviate any biases due to different signals lengths and their histograms were used as input to the three classifiers.

Table 1 tabulates the AM-FM correct classifications success rate for the three classifiers KNN, SOM and SVM and for the five force levels. In addition, the five force level scores per subject were combined with majority voting and the results are also given in Table 1. For the KNN classifier the values provided in Table 1 are for $\mathrm{k}=11$ which gave the best results and for the SOM for a $7 \times 7$ map matrix and an evaluation neighborhood window $3 \times 3$ for the same reason. 
Table 1 AM-FM features correct classifications rate in $\%$ per classifier, per force level and when the five force level scores were combined using majority voting.

\begin{tabular}{|ccccc|}
\hline \hline Force Level & KNN & SOM & SVM & Average \\
\hline $10 \%$ & 52.5 & 55.0 & 67.5 & 58.3 \\
\hline $30 \%$ & 50.0 & 60.0 & 75.0 & 61.7 \\
\hline $50 \%$ & 60.0 & 57.5 & 67.5 & 61.7 \\
\hline $70 \%$ & 52.5 & 55.0 & 65.0 & 57.5 \\
\hline $100 \%$ & 62.5 & 62.5 & 75.0 & 66.7 \\
\hline Average & 55.5 & 58.0 & 70.0 & 61.2 \\
\hline & & & & \\
\hline Combined & 57.5 & 60.0 & 77.5 & 65.0 \\
\hline \hline
\end{tabular}

Table 2 Standard features correct classifications rate in \% per classifier, per force level and when the five force level scores were combined using majority voting.

\begin{tabular}{|ccccc|}
\hline \hline Force Level & KNN & SOM & SVM & Average \\
\hline $10 \%$ & 40.0 & 38.5 & 37.5 & 38.7 \\
\hline $30 \%$ & 40.0 & 47.5 & 42.5 & 43.3 \\
\hline $50 \%$ & 42.5 & 45.5 & 47.5 & 45.2 \\
\hline $70 \%$ & 32.5 & 46.5 & 20 & 33.0 \\
\hline $100 \%$ & 25.0 & 48.5 & 17.5 & 30.3 \\
\hline Average & 36.0 & 45.3 & 33.0 & 38.1 \\
\hline & & & & \\
\hline Combined & 25.0 & 35.0 & 22.5 & 27.5 \\
\hline \hline
\end{tabular}

Best classifier was by far the SVM with average success rate $70.0 \%$ compared to $58.0 \%$ for the SOM and $55.5 \%$ for the KNN classifier. Best force level was the $100 \%$ MVC with average success rate $66.7 \%$ for the three classifiers. Best individual result was $75 \%$ with the SVM classifier at $100 \%$ and $30 \%$ MVC. These results need further investigation and interpretation.

Combining the five force level scores per subject with majority voting improved the average success rate, reaching in the case of the SVM classifier from $70.0 \%$ to $77.5 \%$. Combining all the outputs from all the classifiers gave $62.5 \%$. From the three AM-FM features, the best was the IF feature with average success rate $68.0 \%$ followed by the IA with $55.0 \%$ and the IP with $53.5 \%$.

The same experiment was repeated using the seven time and frequency domain features described in section III. Standard features failed to provide any meaningful results on the given dataset reaching an average correct classification rate of $38.1 \%$ as tabulated in Table 2. These results are well below the $50 \%$ threshold for a two-class problem, which shows the need of a careful extraction and selection of features for this kind of problem and the superiority of the AM-FM algorithm. Feature selection among the standard features did not differentiate significantly the above trend.

\section{CONCLUding Remarks}

In this work it was shown that AM-FM approaches provide new feature sets, which can be used successfully for the classification of SEMG signals with a high success rate; comparable to results obtained when using needle EMG data [3], [4]. The AM-FM features significantly outperformed the standard time and frequency domain features showing that these new features can provide the tool for successful SEMG classification. The results also show that SEMG can be used as a non-invasive alternative to needle EMG for the assessment of neuromuscular disorders.

Additionally in future work, cumulative density functions (CDF) and probability density functions (PDF) can be extracted from the AM-FM representations and used for classification instead of the histograms for an improved classification performance. These along with efficient neural classifiers like the SVM can provide the tools for designing a successful SEMG diagnostic system.

\section{REFERENCES}

[1] Merletti R, De Luca C.J. "New techniques in surface electromyography, in Computer aided electromyography and expert systems," edited by Desmedt J.E., Elsevier, vol. 2, Amsterdam-New York-Oxford, Chapter 9, section 3, pp. 115-124, 1989.

[2] Farina D, Fosci M, Merletti R, "Motor unit recruitment strategies investigated by surface EMG variables," Journal of Applied Physiology, 92, pp. 235-247, 2002.

[3] Abel E.W, Zacharia P.C., Forster A., Farrow T.L., "Neural network analysis of the EMG interference pattern," Medical Engineering and Physics, 1(1): pp. 12-17, 1995.

[4] Christodoulou C.I., Pattichis C.S., Fincham W.F., "A Modular Neural Network Decision Support System in EMG Diagnosis," Journal of Intelligent Systems, Special issue on Computational Intelligent Diagnostic Systems in Medicine, ed. by C.N. Schizas, Volume 8, Nos. 1-2, pp. 99-143, 1998.

[5] Abou-Chadi F.E.Z., Nashar A., Saad M., "Automatic analysis and classification of surface electromyography," Frontiers in Medical Biological Engineering, 1(1)1: pp. 13-19, 2001.

[6] Kaplanis P.A, "Surface Electromyography for the Assessment of Neuromuscular Disorders," Ph.D. Thesis, Kings College, University of London, 2004.

[7] Christodoulou C.I., Kaplanis P.A., Murray V., Pattichis M.S., Pattichis C.S., "Classification of Surface Electromyographic Signals using AMFM Features", CD-ROM Proceedings of the 9th International Conference on Information Technology and Applications in Biomedicine, ITAB 2009, Larnaca, Cyprus, Nov. 5-7, 2009.

[8] Murray V., V. Rodriguez P., Pattichis M.S., "Robust Multiscale AM-FM Demodulation of Digital Images," IEEE International Conference on Image Processing, vol.1, pp. 465-468, Oct. 2007.

[9] Pattichis M.S., Bovik A.C., "Analyzing image structure by multidimensional frequency modulation," IEEE Transactions on Pattern Analysis and Machine Intelligence, vol. 29, no. 5, pp. 753-766, 2000.

[10] Tou J.T., Gonzalez R.C., "Pattern Recognition Principles", AddisonWesley Publishing Company, Inc., 1974

[11] Kohonen T., "The Self-Organizing Map," Proceedings of the IEEE, Vol. 78, No. 9, pp. 1464-1480, Sept. 1990.

[12] Joachims T., "Making large-scale SVM learning practical," In: Schölkopf B, Burges C, Smola A (eds) Advances in kernel methodssupport vector learning, MIT, Cambridge, Chapter 11, 1999. 